\title{
Paper-Digital Meeting Support and Review
}

\author{
Adriana Ispas, Nan Li, Moira C. Norrie \\ Institute for Information Systems \\ ETH Zurich \\ CH-8092 Zurich, Switzerland \\ Email: \{ispas,norrie\}@inf.ethz.ch
}

\author{
Beat Signer \\ Vrije Universiteit Brussel \\ Pleinlaan 2 \\ 1050 Brussels, Belgium \\ Email: bsigner@vub.ac.be
}

\begin{abstract}
Paper notes are still widely used during meetings for the capture and review of information created in meetings. However, personal notes are limited in terms of providing an overview of collaborative work practices and reflecting the evolution of data along successive meeting phases, especially taking into account actions performed on paper and digital media. We propose a solution for the review of meeting data captured along three dimensions of collaboration: paper-digital interaction, private and shared documents as well as pre- and inmeeting information. Based on a general data model, our system enhances the transition between succeeding meeting phases and improves the review of personal and collaborative cross-media meeting material.
\end{abstract}

\section{INTRODUCTION}

Even with remarkable advances in meeting support and review solutions [2], [31], paper-based notetaking continues to be one of the most commonly used work practices in the preparation and capture of information circulated in meetings. Factors such as a high technological overhead or the increased time to accomplish the same tasks may negate the benefits of more sophisticated systems [21]. Natural paper-based notetaking often represents the only means of documenting meetings and further processing information created as part of collaborative in-meeting activities. This is particularly the case in work environments where a meeting support system is not necessary to achieve collaboration goals and thorough meeting records are not required [7].

During co-located collaboration phases, part of the work takes place in shared interaction spaces and may be captured on media such as whiteboards, flipcharts and shared paper documents on tables. Interactive digital whiteboards and tabletop computers are therefore becoming popular in providing support for collaborative work. However, even if this collaborative work is captured, for example by taking pictures of traditional whiteboards or by the software driving the interactive tabletop surface, it can be difficult to maintain an overview for the reviewing of meeting interactions across private and shared interaction spaces. Material is often prepared in advance by individuals and then edited or annotated collaboratively during meetings for later action by the same or another individual. It is therefore necessary to provide support to allow users to easily move information between private and shared spaces, including the transfer between physical and digital media, without requiring major transcription efforts.
Recent technological solutions for integrating paper and digital media, such as Anoto's digital pen and paper technology ${ }^{1}$, facilitate the integration of information captured on paper with digital services and create an opportunity for improved meeting experiences. When using Anoto-enabled notebooks, meeting participants can seamlessly switch between individual work on paper and collaborative work in digitally enhanced shared spaces. A series of experimental systems have proposed mechanisms for transferring paper notes between personal notebooks and shared interactive whiteboard or tabletop surfaces. However, most of these solutions have only marginally addressed issues related to the further management and review of resulting artefacts across the two interaction spaces. We build on previous work for bridging private paper-based and shared digital information spaces based on Anoto technology and propose a solution for digitally reviewing meetings that involve interactions on both paper and digital media along alternative phases of individual and shared work.

To identify system design requirements, we conducted a study on how paper notes are used to document meetings. The resulting system consists of a number of components that enable the data transition between different devices used during private and shared work through successive meeting phases. Features and functionalities provided by the pre- and in-meeting components are used to derive metadata and the organisation of paper and digitally edited data for the postmeeting review. Collaboratively created data is reviewed based on a simple but effective mechanism allowing the facile tracing of cross-media data transitions and flow of data between interaction spaces.

We start in Section II with a discussion of related work. We then present findings of a user study on meeting notetaking practices in Section III. Section IV describes the features of our meeting review solution, highlighting some implementation details. In Section V, we discuss the major design choices made when implementing the system. Concluding remarks are given in Section VI.

\section{RELATED WORK}

A number of collaborative systems have introduced shared interactive surfaces to address shortcomings of tools typically employed for individual work such as laptops. The interactive

\footnotetext{
'http://www.anoto.com/digital-pen-paper.aspx
} 
surface is often used as a mediator for sharing artefacts from personal information spaces [23], [25]. The shared workspace provides a unified view of possibly heterogeneous artefacts and offers better support for structuring and getting an overview of complex information spaces [29], [32] as well as making it possible for multiple users to work simultaneously. Digital tabletops provide further advantages that make them particularly suitable for specific collaborative tasks [18], [24], [30]. Among these, the natural co-habitation of physical and digital artefacts on the table surface makes tabletops particularly suitable for supporting collaboration in cases where personal work is brought into a meeting in the form of paper documents [10]. Other aspects include encouraging communication in a face to face manner, providing better awareness, equitable collaboration through equal access to material and the direct manipulation of information.

A number of existing systems, including Paperizer ${ }^{2}$ [3], Shared Design Space [8], Diamond's Edge [1], the NiCE Discussion Room [9] and DigiPost [12], interconnect personal work recorded on paper with shared information spaces based on Anoto's digital pen and paper technology. Information written on Anoto-enhanced paper with a digital pen can be made available as digital ink data and rendered on tabletop or wall displays. Emphasising an identified need to support both private and shared information spaces, existing work focuses almost exclusively on interaction techniques to encode a user's intention to transfer content from paper to the shared surface. Issues related to managing multiple instances of artefacts and changes made across information spaces or the synchronisation of changes are often not addressed. Furthermore, existing solutions are limited to meeting environments and lack functionality to access in a post-meeting phase collaboratively produced material.

Inspired by previous work [22], [26], Paperizer proposes two sharing mechanisms. Printed content can be "pick-anddropped" or paper sketches can be "sent" synchronously or asynchronously to a digital whiteboard surface. The Shared Design Space project implements the concept of "hyperdragging" [23], enabling the sharing of personal content on a digital tabletop including digital materials stored on a laptop as well as paper content. Heterogeneous sources of information are also supported in the NiCE Discussion Room by enabling interactions with laptop screen captures on an interactive wall. Diamond's Edge uses a technique called "rip to share" to transfer paper content to a multitouch table. After tapping a designated page area, previously circled paper content is sent to the table and further edits inside the selected paper area are mirrored by the shared copy. DigiPost uses Anoto-enabled post-its to achieve a similar proxy approach. Once placed on the touch sensitive tabletop surface, the annotations written on a post-it are synchronised with its digital counterpart and succeeding edits of the physical post-it note will result in updates of the corresponding digital annotation.

There exist various forms of controls to further interact

${ }^{2} \mathrm{http} / / / w w w . m i-l a b . o r g / p r o j e c t s /$ paperizer/ with paper content placed on a shared surface. For example, paper printed buttons to remote control shared content are used in Paperizer, Diamond's Edge or PaperPoint [26]. While an indirection through paper for any editing operation that requires pen input is required in DigiPost and Diamond's Edge, the use of Anoto technology has been extended to interactive surfaces in Shared Design Space, Paperizer and the NiCE Discussion Room, enabling the pen to be used as an input device for both paper and tabletop interactions. This allows further manipulations of shared content by means of top projected buttons controlled with the digital pen as in the Shared Design Space project. In the NiCE Discussion Room, a pen can also be used to manipulate whiteboard overlays that allow switching between layers of heterogeneous content and to interact with content inside the laptop screen capture. The use of the digital pen as a universal input device resulted in an interesting effect in Paperizer, where the touch input is only mentioned for a vertical surface in the environment to which content from the tabletop can be sent.

Shared Design Space and Diamond's Edge deal with evolving content by using overhead projections on the real printout to provide an overview of edits performed on the shared copy of the paper content. For this purpose, $\mathrm{ARTag}^{3}$ markers are attached to each paper page and tracked by a vision system. The drawback of the approach is that the updated content is only available while working with the paper documents in the area covered by the tracking camera. The approach is only effective for simple editing operations. In the case of concurrent private and shared edits by multiple users, more sophisticated collaborative editing and consistency maintaining solutions are required [28]. Furthermore, the updated content is not available for later access outwith the meeting. In the NiCE Discussion Room, the integration of meeting interactions into the "larger context of overarching activities" is mentioned as onc of the identificd requircments and uscrs are reported to have preferred writing on paper rather than on the whiteboard since paper could be taken away after the session. However, the system only addresses the integration of personal work into shared environments during meetings. A solution proposed in Diamond's Edge is to print updated versions of the content and to attach them to notebook pages.

A series of vision-based systems, such as Pictionaire [10], DocuDesk [5] and Designer's Outpost [14], provide similar sets of functionalities. However, interaction is limited to the tracked surface. In addition to already mentioned drawbacks of vision-based solutions, these systems raise some privacy issues given that personal content can only be manipulated on the interactive surface [10]. On the other hand, systems belonging to this category have proposed further support for content management. Pictionaire introduces the concept of "collection containers" to provide some sort of content organisation and review based on a timeline. Similarly, the workspace can be bookmarked and the bookmarks can be reviewed based on a timeline in Designer's Outpost. Pictionaire further supports the

\footnotetext{
${ }^{3}$ http://www.artag.net
} 
recording and later replay of collaborative sessions. DocuDesk introduces the "task rehydration" feature that enables resuming previous workspace configurations comprising both paper and digital documents. For this purpose, documents displayed on the tabletop surface need to be explicitly linked by the user. In the revived workspace configuration, digital counterparts of the paper documents created by the vision system are presented, enabling comparisons with possibly updated paper versions. A limitation of the review functionality is that privacy aspects are not taken into account when constructing views of the collaboration data [25], [31]. Therefore, all participants have access to any tracked and recorded document, even if they are not meant to be permanently shared with other participants but merely shown during a phase of collaboration. Some review functionality is provided on the table surface in the same setup that is used for the capturing of content but not in the privacy of personal computers. Furthermore, different participants may have different perceptions of interesting content [15]. Among the described systems, only Pictionaire proposes variable views.

Related to our work, CoScribe [27] has proposed a series of interaction mechanisms to integrate and later enhance the review of complementary paper and digital material. However, related documents are added to the proposed interactive graph visualisation only if actively linked through association gestures. Furthermore, approaches for differential visibility of collaborative material apply only to annotations and tags on printed documents.

As highlighted in this section, most of the existing approaches focus on the collaborative issues during a meeting. These solutions pay less attention to the post-processing of information that has been collaboratively generated within a meeting. As a result, it is often not possible to track the transition of information between private and shared information spaces or across different types of media as part of a postmeeting review process.

\section{MEETING Notetaking StUdY}

Systems that deal with the integration of private work captured on paper into collaboration environments often provide limited support for the management and review of generated content. On the other hand, existing meeting support systems create highly accurate representations of in-meeting interactions based on multimodal recordings and multiple views of the recorded content can be consulted by means of integrated meeting browsers. However, the use of these systems in traditional working environments is not widespread due to the high complexity of locating content of interest from large amounts of recorded streaming data [13]. We performed a study on notetaking in meeting environments where notetaking is the primary means of documentation to understand what issues affect in-meeting collaboration and how to best make data available for post-meeting reviews. Our goal was to identify requirements for a meeting support and review solution suited to environments with incidental notetaking.
Extensive details about the method, the recruiting process and the results of our study are presented in a previous publication [11]. The study revealed 7 categories of paper notes based on how they supported post-meeting activities. Notes taken with the purpose of supporting work in progress were the most often recorded and amounted to $37 \%$ of the total notes. They were integrated into digital deliverables a short time after the meeting through a process of updating, restructuring and modifying their content. $21 \%$ of the notes represented todos and reminders. These were typically underspecified and users provided additional details to create entries within digital calendars or similar tools in a postprocessing step after the meetings took place. $6 \%$ of the notes were meant to temporarily record information that is not necessarily of interest for the participant, but which was meant to be later forwarded to other colleagues. Another $8 \%$ of the notes represented information that was classified as potentially relevant in the future. The participants declared that they generally experience difficulties in managing this category of notes as they tend not to be encountered in the future, a phenomenon described in Lin et al. as "out of sight, it is very likely out of mind" [17]. The rest of the notes comprised metadata $(6 \%)$, means of diverting attention $(2 \%)$ and notes declared as irrelevant $(20 \%)$. In the latter case, participants reported that they would take these notes "just in case" but most likely never use them.

We further report on a series of additional aspects that were analysed during the final semi-structured interview phase of our study. We were interested in finding what kind of material is prepared and brought into a meeting by the participants. We also made inquiries regarding the parallel use of paper and digital documents and whether participants experienced any difficulties in managing the combination of paper notes and other information sources after the meetings. Furthermore, we wanted to investigate different approaches to managing evolving information through successive meetings. Finally, we wanted to learn in which manner personally created notes are shared with other participants.

Three predominant types of meeting material were prepared in advance by participants. First, users declared that they make notes in their personal notebooks about issues to be discussed during the meeting. In a few cases, these notes were interleaved with empty placeholders for information to be added during the meeting. A second category comprises printed documents, such as slide handouts, diagrams or document drafts, that are brought into a meeting by a participant to support the discussions. Electronic documents such as slides or wikis containing the meeting agenda represented a third dominant category. In the case that the discussion was supported by publicly shown digital documents, the owner typically performed the changes directly in the digital document version. This was cxplaincd by the bencfit of increased awareness of other participants. The rest of the participants either took paper notes in their personal notebooks or no notes at all. In the case that the discussion was supported by printed documents, users preferred to annotate the received copy instead of writing in a 
personal notebook. Participants were rather fuzzy in terms of their approach to managing annotated documents. These were typically kept in printed annotated form for later reference, without any digital transcription. In a few cases, participants mentioned that they filed these paper documents within or in the vicinity of their notebooks, particularly a short time after the meeting. However, participants also reported that these annotated documents were often misplaced in the long term.

Normally, notes taken during a meeting were not completed with supplemental details after the meeting and there was a lack of managing evolving content. An exception were the participants who intentionally left empty placeholders in their pre-meeting notes and filled in information during the meeting. Only one participant managed evolving content by rewriting the updated content as a new entry in their notebook. The result can be explained by the preference to produce more refined versions of meeting notes in a digital form as mentioned earlier in the case of notes supporting work in progress.

Participants reported that they normally do not share notes by physically passing around their notebooks. They mentioned that the notes were too sketchy to be forwarded in the form in which they appear in the notebook and also, for reasons of privacy, they did not want to make the entire content of their notebooks available. Notes taken to inform others were usually communicated verbally. In a few cases, participants used email communication after the meeting. In one meeting, participants used post-its to comment on a colleague's presentation and handed them to the presenter after the meeting. When asked, the participants expressed an interest in a service that could potentially allow them to easily share occasional notes taken for somebody else's interest.

We asked the participants which aspects of their meeting material management practice could accommodate improvements. Three aspects were associated with the highest potential for improvements. First, the questioned people mentioned that they were not able to find a single tool that could manage all of their information; a problem that has been investigated in the field of personal information management for quite some time. Second, they expressed their dissatisfaction about not having found a way to organise and get an overview of all their paper material including printed documents as well as handwritten notes. Third, it was reported that it would be good to have a record of related content in the case that notes referred to other documents. For example, handwritten notes might be associated with the points in a printed agenda that are not explicitly copied into the notebook.

Based on the observation that participants processed less urgent notes mostly when randomly encountered "in their way", our previous recommendation for the processing of paper notes was to focus on reminder functionality [11]. In this paper, we address the case of information captured as paper notes that needs to be refined into digital representations in situations when users are required to actively seek information of interest such as the case of notes supporting work in progress. A mechanism to overview the entire set of information, possibly stored as a combination of paper notes and other resources, as well as a solution to filtering relevant information from heterogeneous notes in a paper notebook are required. In the next section we present our solution for the latter case.

\section{Note-BASEd MEeting SupPort AND REVIEW}

We start this section by listing the requirements of our meeting support and review system and then describing the main features of the system. In the third part, we provide some implementation details.

\section{A. System Requirements}

The results from related work and our own study lead to the following main requirements.

Paper-based notetaking as primary documentation: Our study participants were reluctant to change their meeting documentation behaviour [11]. They also declared that they would accept changes imposed by a meeting support system only if the benefits clearly outweigh additional efforts. It became obvious that any kind of support should not require that meeting participants use the system instead of their habitual documenting approach. The system should rather complement their preferred paper-based documentation work practices. Lin et al. [17] recommend that pen and paper are provided for recording information when it is triggered, but emphasise that digital solutions are typically more suitable for later transfer, maintenance, reference and archiving phases in the information management cycle. Therefore, our aim was to allow participants to primarily refer to notes and use the meeting support system to enhance the sharing of information both during and after meetings.

Cross-media transitions between private and shared spaces through different meeting phases: It is obvious that different situations may require different degrees of refinement of content brought into meetings to support collaboration. In some cascs, it may suffice to simply jot down some notes, while in others digital documents may be a more appropriate representation. As mentioned in Section II, several factors result in less efficient collaboration when information that has been captured on paper has to be integrated into shared environments. The collaboration supported by a user's physical notebook can be rather unnatural, lacks equalitarian input and equal access to material. Transcription is normally required to make the information available on blackboards or other shared spaces. In Section III, we discussed that printing prepared digital materials to support in-meeting collaboration leads to a fragmentation of notes into multiple paper resources. Similarly, digital projections result in a separation of notes from the content to which they refer. Therefore, a mechanism to make both paper and digital private material easily available in shared spaces is required. In addition, we think that premecting phases should also be addressed. Participants should not only be able to prepare material when physically located in the vicinity of the shared spaces, but also on their personal workstations as part of a pre-meeting phase. The distribution of information and easy post-meeting access to information generated in shared spaces should also be ensured. Furthermore, a 
mechanism to interrelate pieces of information across private and shared spaces for documenting inter-space transitions and dependent edits is required and participants should be able to correlate their notes with material from the shared spaces. As reported in our study [11], notes have different uses that can only be identified by the user. Therefore, participants should be given the possibility to control and actively enforce the process of interrelating parts of their notes with external material.

Granular content sharing and post-meeting data ownership: Different categories of notes occur in notebooks. It is therefore important that users are able to selectively share parts of their paper notes. The privacy of a user's notes needs to be given further attention in post-meeting phases. In a meeting review, several levels of access to material managed in shared spaces should be enforced. The owner of the shared material should be able to control who gets what type of access to their data after the meeting. This could encourage the sharing of material in the first place and support in-meeting collaboration.

Quick review of paper-digital material that has been handled in meetings: Meetings can generate a lot of paper and digital data. When using Anoto technology, handwritten information is captured as low level ink data. In addition to ensuring that relations between artefacts are appropriately captured and enforced in a meeting review component, appropriate levels of granularity in presenting data and intuitive entry points for navigating captured material should be provided [31]. Various approaches of dealing with low granularity levels presented by streaming data have been proposed. Ju et al. [13] propose a timeslice-based model to reduce the complexity of recorded data. In Teamspace, indexes based on interactions with artefacts are recommended to ease retrieval of recorded material [7]. In the case of ink data, Pimentel et al. [20] propose a review solution based on replaying pen-based interactions and address the problem of low granularity by constructing intermediary views. As we will describe in the next subsection, we opted for snapshots containing intermediary states of paper notes snippets and annotated digital documents processed in the shared space to organise data for the review phase. Participants can obtain a quick overview of the collaboration by inspecting captured collaborative artefacts and identify entry points for filtering material of interest from their notebooks. A similar conclusion is reached by Czerwinski and Horvitz [4] who recommend snapshots of activities in the form of still images to review computing events.

\section{B. Paper-Digital Meeting Assistant}

The interaction design of our system is logically separated according to the pre-meeting, in-meeting and post-meeting phases. The pre-meeting component provides the required functionality to prepare upcoming meetings. The in-meeting component supports co-located collaboration based on an topprojected and Anoto-enabled tabletop system that can be controlled via digital pen input. Participants may collaborate while seated around the tabletop and, at the same time, take notes in their private notebooks that can be comfortably placed outside of the projected tabletop interface as shown in Figure 1. The post-meeting component provides support for reviewing paper notes and digital documents that have been worked on during a meeting. We will describe the functionality of each of these three components in more detail.

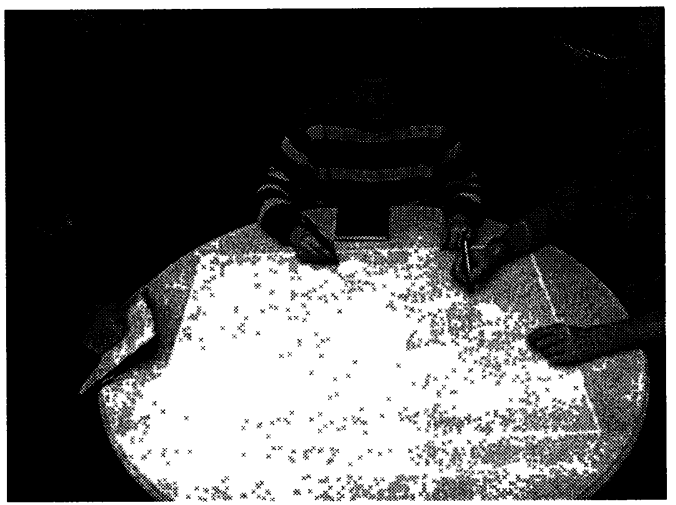

Fig. I. In-meeting interaction

Meeting participants are provided with support to collect and upload both paper and digital materials to a virtual document space before a meeting. The uploaded material will be accessible for interactions in the shared interaction space during a specific meeting chosen from a dropdown list showing all upcoming meetings. To create a new meeting event, a user has to provide the relevant meeting details including a title, a list of topics and the participants as shown in Figure 2. Similarly, new user profiles can be created by providing a name, a password and a list with the identifiers of associated digital pens. The information defined in a user profile will be used to control access during in-meeting and post-meeting phases. Furthermore, in-meeting interactions will be associated with individual users based on the unique digital pen identifiers.

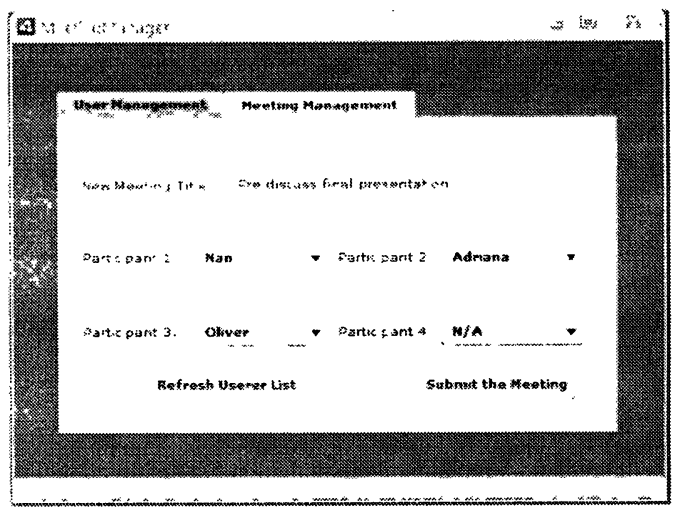

Fig. 2. Meeting event creation

To upload paper content, participants are required to use Anoto-enabled notebooks. Previously written paper notes can be selected via a pen-based cropping gesture similar to the 
approach used in PapierCraft [16], as shown in Figure 3a. Selected parts of notebook pages are transformed into digital representations and stored in the virtual document space. The Anoto digital pens are used in streaming mode so that paperbased user actions are continuously interpreted and feedback can be provided on the user's personal computer screen. Any selected paper content is represented as a virtual note and a thumbnail of it appears on the central cover flow widget of the application window as highlighted in Figure 3b. Digital documents can be selected via a file chooser component. The first page of a selected document is shown as a thumbnail picture in the cover flow list of prepared documents in a similar manner to Figure $3 b$.

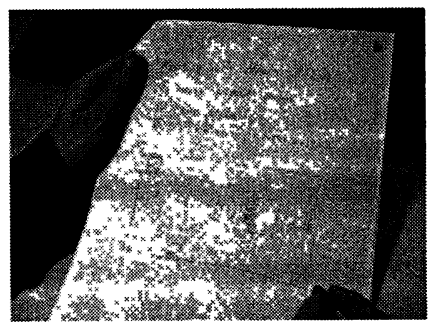

(a) Select note content

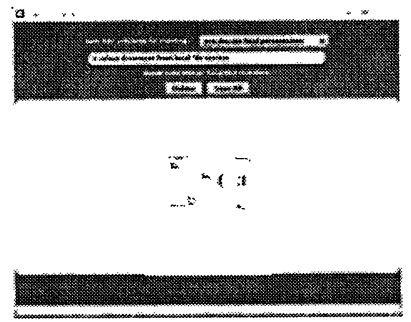

(b) Display note thumbnail
Fig. 3. Collection of paper notes

The tabletop application user interface shown in Figure 4 provides several elements for organising meeting material. In the centre, a set of buttons placed on a rotating Wheel of Fortune are easily accessible by all participants and provide functionality to create workspace snapshots, reestablish previous workspace layouts or save and delete documents.

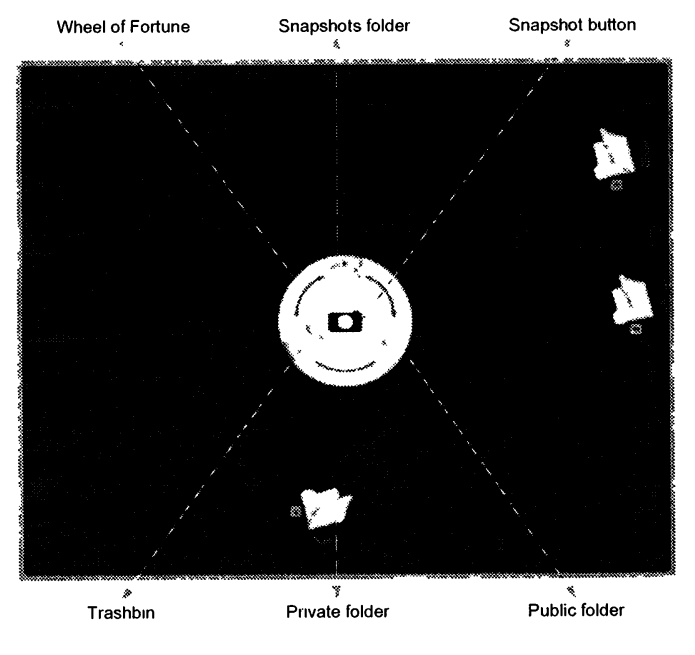

Fig. 4. Tabletop user interface

By touching the Snapshot button with the pen, the current tabletop configuration is capturce and storcd as a snapshot. A previously captured workspace snapshot can be loaded to the tabletop by touching the Snapshots folder and dragging the corresponding thumbnail representation from a cover flow interface that contains all of the snapshots taken in the current meeting session as shown in Figure 5. The dropping of documents into the Public folder will later make them accessible to all participants in the meeting review phase. Furthermore, any document that is dropped into the Trashbin is permanently deleted. Paper notes and digital documents that have been prepared and uploaded in the pre-meeting phase are accessible during a meeting via each participant's Private folder. The position of a private folder can be changed by simply dragging and dropping the folder with the digital pen. Note that the folders will reorient themselves when repositioned by applying an auto-orientation technique similar to MERL's DiamondSpin ${ }^{4}$.

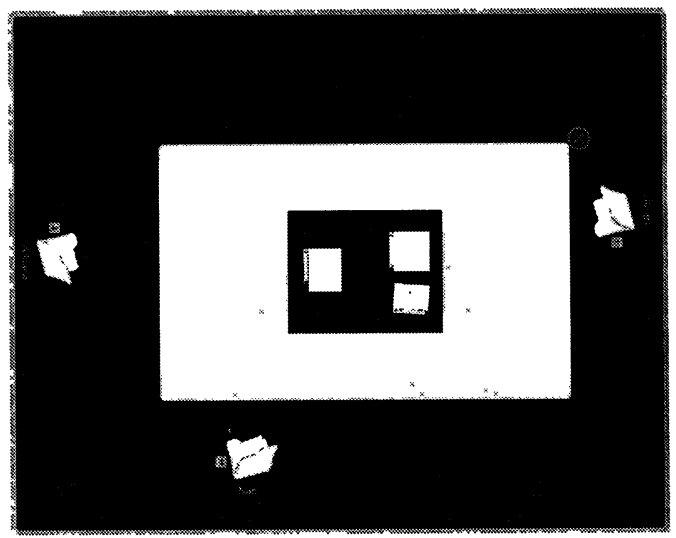

Fig. 5. In-meeting review of workspace snapshots

Paper notes excerpts are represented on the tabletop user interface as virtual notes containing a digital counterpart of the original paper note. Digital documents are represented as virtual physical books that can be consulted through a simulated 3D page turning effect. Pen input on both a virtual note and individual pages of a virtual book results in penbased annotations of the original content. In the case of notes, the new pen strokes update the digitised note content, while annotations on a virtual book page are associated with each page in the form of overlay information.

A series of further editing operations are available for both content types when moved into the shared area of the tabletop. These operations are provided through decentralised toolbars associated with each document or note and through contextual menus triggered by a double tapping gesture, as shown in Figure 6. The toolbars include action items to save a public document in a user's private folder, delete the document, change the colour palette and switch between different editing modes. The available pen-based modes of interaction are freehand sketching to update a virtual note or annotate digital document pages, zooming, rotating and moving the corresponding document. The contextual menus provide copy and paste, delete and deselect operations of previously selected parts of virtual notes as well as digital document annotations. The pen input is switched to selection

\footnotetext{
${ }^{4}$ http://www.merl.com/projects/diamondspin/
} 
mode after having touched a dedicated toolbar item. New content can be created on the tabletop in the form of new virtual notes. A virtual note is created by drawing a rectangle with the pen as shown in Figure 7a.

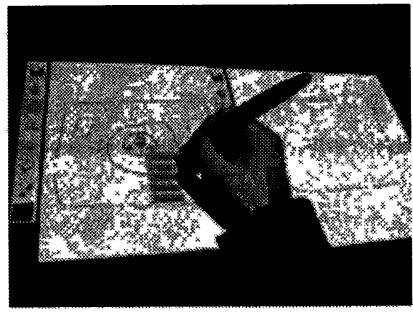

(a) Copy selected strokes

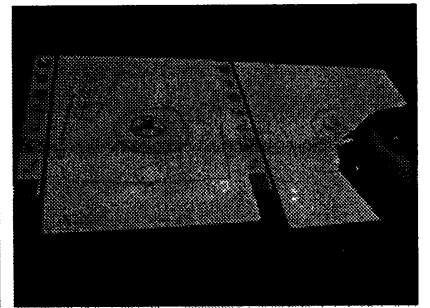

(b) Paste selected strokes
Fig. 6. Copy/paste operation

As soon as the user lifts their pen, an empty virtual note appears on the tabletop surface at the same position and with the corresponding size as highlighted in Figure 7b. By applying the auto-orientation technique mentioned earlier, the new note automatically faces its creator.

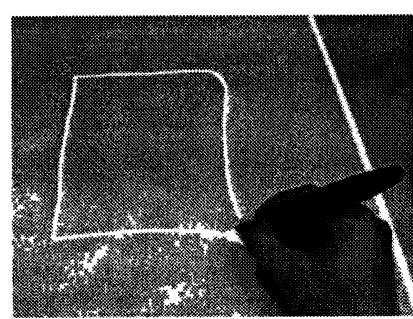

(a) Draw rectangle

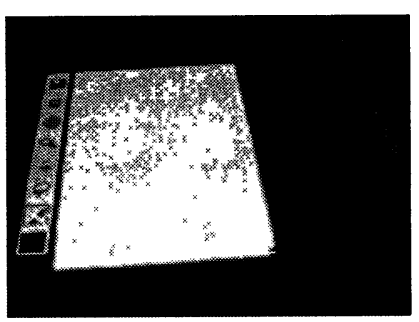

(b) Empty virtual note
Fig. 7. Virtual note creation

Virtual notes created on the tabletop are by default public. A participant may claim the ownership of a public note by dragging the note to their private folder or by invoking the corresponding action provided by the associated toolbar widget. Documents placed in a participant's private folder can only be accessed by their owner. When double tapping the folder with one of their registered pens, a cower flow widget containing thumbnails of the available documents opens and documents can be drag-and-dropped to the shared space. Once documents are made available in the shared space, they can be edited by other users. However, in the post-meeting review, they will have restricted access unless the owner explicitly shared them with other users by dropping them into the Public folder in the centre of the tabletop surface.

Users may also transfer parts of their notes onto the tabletop surface during the meeting. By using the same cropping gesture mentioned for the pre-meeting component, selected notebook content is made available as a virtual note in a users's private folder. Icons attached to the folder inform the user about the status of their interactions on paper.

Selected paper notes can also be directly made available on the tabletop without any indirection through a private folder, when the selection is performed by using a circling gesture as highlighted in Figure 8. The note appears on the tabletop at the position indicated by a double tap gesture performed successively to the circling selection operation.

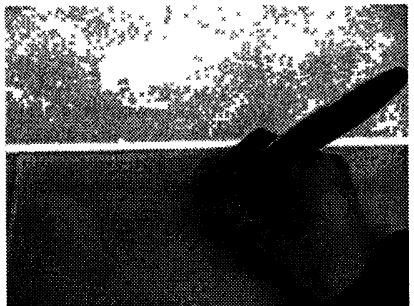

(a) Circle paper note

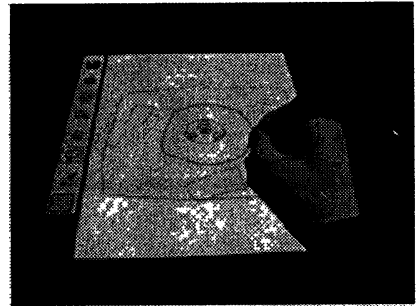

(b) Paste selected paper content
Fig. 8. Copy a paper note directly to the tabletop

As mentioned carlier, the use of private and public folders to change the ownership of public material created on the tabletop during the meeting and private material created by individual participants within their private space, is reflected in the post-meeting review. If the ownership has not been changed, stored information about a document's creator is used. Meeting material can be reviewed by using the same cover flow interface approach. Users may browse through collections of workspace snapshots as shown in Figure 9. Virtual notes and physical books representing digital documents are highlighted in different colours according to their ownership. Artefacts owned by the currently logged in user are highlighted in pink, public artefacts are highlighted in yellow and artefacts pertaining to other users, and therefore inaccessible, are not highlighted. When a user clicks on a highlighted thumbnail, the note or document is enlarged to expose more details.

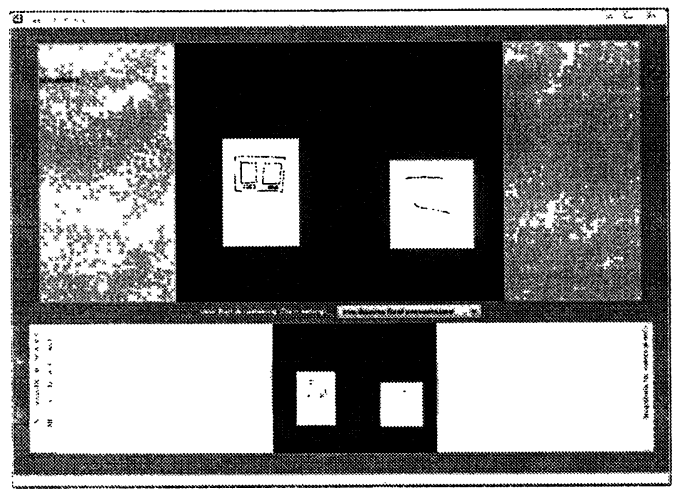

Fig. 9. Review based on snapshots

The cover flow allows users to obtain a quick overview of all collaborative artefacts and the highlighting helps users quickly realise what content they can access. It may be that not all documents are comprised within snapshots. A separate view allows users to browse through all non-snapped documents for which they have access rights as shown in Figure 10.

A final review functionality allows users to get quick access to updated versions of previously shared paper notes. When a user taps the pen within the extent of selection marks made 


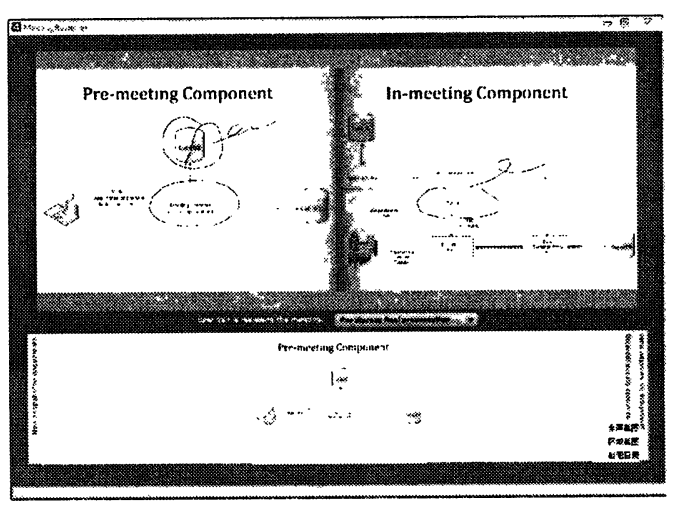

Fig. 10. Review of documents handled on the tabletop

on paper when sharing the note, the corresponding virtual note and all snapshots containing different versions of the note are retrieved and shown as thumbnail images in a cover flow widget in a third view of the post-meeting component as shown in Figure 11. Each shared paper note may be associated with several virtual notes, depending on whether snapshots have been created or the virtual notes have been deleted in the meanwhile. The corresponding virtual notes are highlighted in red when shown as part of a captured snapshot. The snapshots can be interacted with as described previously.

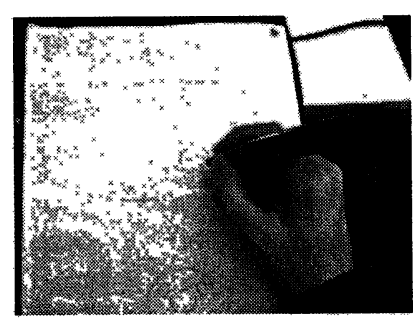

(a) Tap inside notebook selection

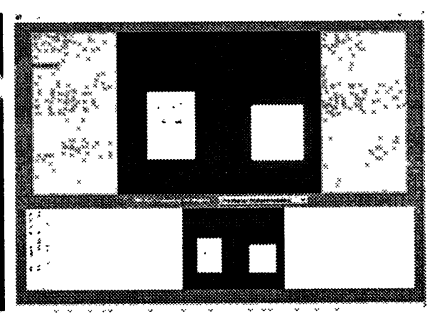

(b) Browse artefacts containing note
Fig. 11. Finding the updated state of a shared note

\section{Implementation}

Figure 12 shows the main components of our system. The system consists of three modules, one for each meeting phase, and a central database module that manages information about meetings, users and documents in the virtual document space. The database module is the mediator that allows data captured in different meeting phases to be accessible across phases as indicated by the blue arrows.

The general data model with the main entities and relationships is shown in Figure 13 using UML notation. Administrative data is recorded as instances of the User and Meeting classes which capture user profile and meeting event details. In the case of users, details about their name, password and the unique pen addresses are stored. A meeting is described by its title and a list of topics as well as participating users. As described earlier, the information is entered through the pre-meeting application user interface.

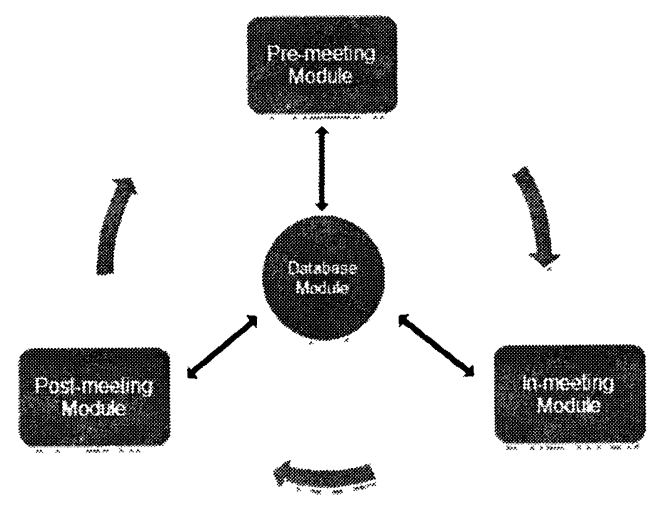

Fig. 12. System components

To be able to manage edits performed on the extent of various types of artefacts handled in the shared space, we introduced the high level abstractions of Documents and Notes. A Document models different types of content that can be displayed and manipulated on the tabletop surface, including virtual notes or PDF documents. Pen-based annotations made on the extent of a virtual note or digital document page are represented as Notes which are associated with the corresponding Document instance. A Note is composed of all the edits made on the extent of a Document page, such as traces containing collections of successive pen coordinates in the case of Anoto technology. A document is created by a user and is, by default, accessible to its creator. Access rights can be granted to other users, for example in the case of virtual notes created on the tabletop by a participant and devised public by the system or documents dropped into the Public folder or personal folders of other participants than the creator, as modelled by the accessibleTo association.

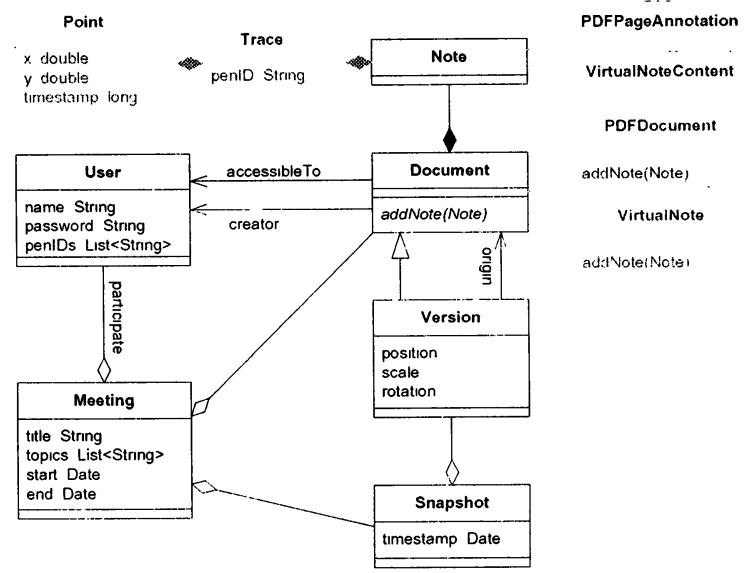

Fig. 13. Data model

Workspace snapshots are modelled as Snapshots which are associated with a specific meeting and refer to a set of document versions. When a new snapshot is created, the statc of cach document placed on the tabletop at that specific 
moment is captured with an instance of the Version class, which is associated with the snapshot object and original document. A document version maintains information about a document's representation on the tabletop and all contained edits at the moment of the snapshot. If a snapshot has been reconstructed, the document versions can be further edited, moved and resized. A subsequent snapshot will create new document versions.

To address differences in the manipulation of various types of documents and their annotations, extensions of the two general Document and Note classes can be implemented. In Figure 13, we show the extensions that we made to handle virtual notes and PDF documents in grey. The two specific implementations of the Document class are VirtualNotes and PDFDocuments. Pen-based annotations are represented by the VirtualNoteContent and PDFPageAnnotation extensions of the Note class. A PDFDocument can have zero or multiple PDFPageAnnotations associated to it. In contrast, a virtual note can have at most one associated virtual note content. Such document specific cardinality constraints over the Document-Note association are specified in the corresponding Document subclass implementations as indicated by the abstract addNote method.

Pen input on both paper and the tabletop surface covered with Anoto pattern is handled by the iPaper framework [19]. For the tabletop surface used during the in-meeting phase, we used an inexpensive solution consisting of a large paper sheet printed with Anoto pattern placed on a regular circular table under a protective glass layer. An in-meeting application user interface implemented in Adobe Flex ${ }^{5}$ is overhead projected onto the table surface and, through appropriate calibration, pen input interpreted by the iPaper framework is mapped to corresponding manipulations of underlying projected content. To implement multitouch operations such as zooming, rotating and moving content on the table surface, which require fast transmissions in real time, the pen input is mapped to TUIO $^{6}$ messages. These messages are transmitted to the application driving the tabletop user interface and parsed into multitouch gestures. We use a modificd TUIO cursor profilc to also transmit information about pen identifiers and timestamps as part of the TUIO messages. These were used for operations on the tabletop requiring user identification, such as the autoorientation feature. The database module uses a $\mathrm{db} 4 \mathrm{o}^{7}$ object database engine in client/server modc.

\section{Discussion}

Our approach to reviewing collaboration data relies on a series of features introduced in the pre- and in-meeting interaction. We use virtual notes and digital document page annotations to cluster ink data representing interactions in the shared space. Workspace snapshots further group related content of potentially heterogeneous types. Notes and snapshots introduce two levels of coarser granularity in reviewing

\footnotetext{
${ }^{5}$ http://www.adobe.com/products/flex/

${ }^{6} \mathrm{http}: / / \mathrm{www}$. tuio.org/

${ }^{7}$ http://www.db4o.com/
}

meeting interactions, as compared to replaying individual operations [20]. Appropriate privacy levels in reviewing meeting material are ensured through private and public folders used to adapt ownership metadata. Furthermore, a browsing approach based on cover flow widgets allows uscrs to quickly identify possible information of interest among the captured data. Our choice follows recommendations made for meeting support systems, according to which the browsing interface has to be simple and provide quick overview with low level effort required for the users [31].

The proposed solution is meant to support participants in processing meeting material, especially in cases driven by a specific task such as producing an outcome artefact. These tasks require an overview of relevant material. In our system, an overview of paper and digital material handled in the shared space during meetings is constructed, along with means of filtering and discovering entry points into the heterogeneous content of paper notebooks. In addition to supporting actively looking for specific relevant content, the mechanism may create opportunities to casually notice or increase the ability to remember other useful information, as suggested in our previous publication [11]. Since our study has shown that documentation material is most likely modified and a digital version of the same content would not necessarily be of use, we did not focus on supporting the creation or edits of digital artefacts based on content extracted from the overview.

Whittaker et al. [31] suggest that one of the reasons why multiple meeting browsers have not gained popularity might be having "failed to provide an appropriate level of abstraction to allow users to strategically focus on important parts of the meeting". The abstractions we propose for structuring and presenting meeting material for review are notes made on documents and snapshots. They reflect users' assessments on the relatedness of content. Edits can be performed on the extent of the same virtual note or document page to modify or add related content. New virtual notes can be created when unrelated content has to be dealt with. By default, notes reflect the final result of edits performed during collaboration to avoid generating too many or irrelevant stages of the collaboration in the final review and enforce the efficiency of the navigation. Intermediary stages of the content can be captured as part of snapshots when necessary. Snapshots provide a way of saving intermediary work states and revive them on the tabletop, but at the same time group notes that are related temporally in that they were handled around the same time. In the case of snapshots navigation, the reviewing approach combines a timeline with semantic grouping enforced by the user.

The approach used for capturing and organising meeting data is further derived from users' assessment of relevancy. In Teamspace [7], interactions with artefacts such as slide transitions were considered relevant markers in browsing meeting material. We attributed a similar role to shared material represented by paper snippets transferred to the shared space and documents brought into a meeting. Given the mixed character of paper notes that users normally take, the fact that only shared notes are presented in the review introduces a 
filtering mechanism based on what was considered relevant for the discussion at a specific moment.

Being derived from users' assumptions of relatedness and relevancy, the approach to overviewing meeting data is likely to be more effective, as also pointed by Fass et al. [6]. However, the same authors mention that users are likely not to spend time on documenting interactions when focusing on other activities. Ju et al. [13] introduced the idea of implicit and explicit captures used in combination to cope with this issue. In our opinion, a form of implicit capture would have neglected our initial goal of providing quick and effective overview of collaboration data. Therefore, we chose to make a compromise on the assumption that if users do take the time for additional actions such as uploading documents into the system or generating snapshots, then these would most likely represent relevant data. At this stage, we have performed an informal evaluation of the current system and, overall, users were positive about its features. We are currently planning an extended user study of the presented system.

\section{CONCLUSION}

Previous work investigated various technological approaches for integrating private paper-based information within shared interaction spaces. We propose a system that, based on a series of features introduced to support the different meeting phases, provides means to review both collaborative and personal meeting data and prepare meeting material to be shared, in addition to supporting in-meeting interactions between private and shared information spaces. The system was informed by a user study on incidental paper-based notetaking. Meeting data is managed based on a general data model that allows us to enforce granularity and access levels while presenting data in the review phase.

\section{REFERENCES}

[1] M. Bernstein, A. Robinson-Mosher, R. B. Yeh, and S. R. Klemmer. Diamond's Edge: From Notebook to Table and Back Again. In Extended Abstracts of UbiComp '06, Orange County, USA, September 2006.

[2] M.-M. Bouanrane and S. Luz. Meeting Browsing State-of-the-art Review. Multimedia Systems, 12(4-5):439-457, March 2007.

[3] P. Brandl. M. Haller. J. Oberngruber. and C. Schafleitner. Bridging the Gap Between Real Printouts and Digital Whiteboard. In Proc. of AVI '08. Napoli, Italy, May 2008.

[4] M. Czerwinski and E. Horvitz. An Investigation of Memory for Daily Computing Events. In Proc. of $\mathrm{HCI}^{\circ}$ (2), London, England, September 2002.

[5] K. M. Everitt, M. R. Morris, A. B. Brush, and A. D. Wilson. DocuDesk: An Interactive Surface for Creating and Rehydrating Many-to-Many Linkages among Paper and Digital Documents. In Proc. of Tabletop '08, Amsterdam, The Netherlands, October 2008.

[6] A. Fass, J. Forlizzi, and R. Pausch. MessyDesk and MessyBoard: Two Designs Inspired by the Goal of Improving Human Memory. In Proc. of DIS 02 , London, England, June 2002.

[7] W. Geyer. H. Richter, and G. D. Abowd. Towards a Smarter Meeting Record-Capture and Access of Meetings Revisited. Multimedia Tools and Applications, 27(3):393-410, December 2005.

[8] M. Haller, D. Leithinger, J. Leitner, T. Seifried, P. Brandl, J. Zauner, and M. Billinghurst. The Shared Design Space. In Proc. of SIGGRAPH '06 Emerging Technologies, Boston, USA, July-August 2006.

[9] M. Haller, J. Leitner, T. Seifried, J. Wallace, S. Scott, C. Richter, P. Brandl, and A. Gokcezade. The NiCE Discussion Room: Integrating Paper and Digital Media to Support Co-Located Group Meetings. In Proc. of $\mathrm{CHI} \%$ (), Atlanta, USA, April 2010.
[10] B. Hartmann, M. R. Morris, H. Benko, and A. D. Wilson. Pictionaire: Supporting Collaborative Design Work by Integrating Physical and Digital Artifacts. In Proc. of $\mathrm{CSCW}$ '10, Savannah, USA, February 2010.

[11] A. Ispas, B. Signer, and M. C. Norrie. A Study and Design Implications for Incidental Notetaking with Digital Pen and Paper Technologies. In Proc. of $\mathrm{HCI}$ '10, Dundee, Scotland, September 2010.

[12] H. Jiang, R. B. Yeh, T. Winograd, and Y. Shi. DigiPost: Writing on Postits with Digital Pen to Support Collaborative Editing Tasks on Tabletop Displays. In UIST 'O7 Posters, Newport, USA, October 2007.

[13] W. Ju, A. Ionescu, L. Neeley, and T. Winograd. Where the Wild Things Work: Capturing Shared Physical Design Workspaces. In Proc. of CSCW'04, November 2004.

[14] S. R. Klemmer, K. M. Everitt, and J. A. Landay. Integrating Physical and Digital Interactions on Walls for Fluid Design Collaboration. HumanComputer Interaction, 23(2):138 -213, April 2008.

[15] J. A. Landay and R. C. Davis. Making Sharing Pervasive: Ubiquitous Computing for Shared Note Taking. IBM Systems Journal, 38(4):531$550,1999$.

[16] C. Liao, F. Guimbretière, and K. Hinckley. PapierCraft: A Command System for Interactive Paper. In Proc. of UIST '05, Seattle, USA, October 2005.

[17] M. Lin, W. G. Lutters, and T. S. Kim. Understanding the Micronote Lifecycle: Improving Mobile Support for Informal Note Taking. In Proc. of $\mathrm{CHI}$ '04, Vienna, Austria, April 2004.

[18] P. Marshall, E. Hornecker, R. Morris, N. S. Dalton, and Y. Rogers. When the Fingers Do the Talking: A Study of Group Participation with Varying Constraints to a Tabletop Interface. In Proc. of Tabletop '08, Amsterdam, The Netherlands, October 2008

[19] M. C. Norrie, B. Signer, and N. Weibel. General Framework for the Rapid Development of Interactive Paper Applications. In Proc. of COPADD '06, Banff, Canada, November 2006.

[20] M. G. Pimentel, C. Prazeres, H. Ribas, D. Lobato, and C. Teixeira Documenting the Pen-based Interaction. In Proc. of WebMedia '05, Pocos de Caldas - Minas Gerais, Brazil, December 2005.

[21] C. Plaue and J. Stasko. Presence \& Placement: Exploring the Benefits of Multiple Shared Displays on an Intellective Sensemaking Task. In Proc. of GROUP '09, Sanibel Island, USA. May 2009.

[22] J. Rekimoto. Pick-and-drop: A Direct Manipulation Technique for Multiple Computer Environments. In Proc. of UIST '97, Banff, Canada, October 1997.

[23] J. Rekimoto and M. Saitoh. Augmented Surfaces: A Spatially Continuous Work Space for Hybrid Computing Environments. In Proc. of CHI '99, Pittsburgh, USA, May 1999.

[24] S. D. Scott, K. D. Grant, and R. L. Mandryk. System Guidelines for Co-located, Collaborative Work on a Tabletop Display. In Proc. of ECSCW '03, Helsinki, Finland, September 2003.

[25] C. Shen, K. Everitt, and K. Ryall. UbiTable: Impromptu Face-toFace Collaboration on Horizontal Interactive Surfaces. In Proc. of UbiComp' ()3, Seattle, USA, October 2003.

[26] B. Signer and M. C. Norrie. PaperPoint: A Paper-based Presentation and Interactive Paper Prototyping Tool. In Proc. of TEI '07, Baton Rouge, Louisiana, February 2007.

[27] J. Stcimle, O. Brdiczka, and M. Mühlhäuser. CoScribe: Integrating Paper and Digital Documents for Collaborative Knowledge Work. IEEE Trans. on Learning Technologies, 2(3):174-188, July-September 2009.

[28] M. Sugimoto, K. Hosoi, and H. Hashizume. Caretta: A System for Supporting Face-to-face Collaboration by Integrating Personal and Shared Spaces. In Proc. of $\mathrm{CHI}^{\circ}$ (04, Vienna, Austria, April 2004.

[29] P. Tuddenham, I. Davies, and P. Robinson. WebSurface: An Interface for Co-located Collaborative Information Gathering. In Proc. of ITS 'O9, Banff, Canada, November 2009.

[30] J. R. Wallace and S. D. Scott. Contextual Design Considerations for Co-located, Collaborative Tables. In Proc. of Tabletop '08, Amsterdam, The Netherlands, October 2008.

[31] S. Whittaker, S. Tucker, K. Swampillai, and R. Laban. Design and Evaluation of Systems to Support Interaction Capture and Retrieval. Personal and Ubiquitous Computing, 12(3):197-221, March 2008.

[32] D. Wigdor, H. Jiang, C. Forlines, M. Borkin, and C. Shen. WeSpace: The Design, Development and Deployment of a Walk-up and Share Multisurface Visual Collaboration System. In Proc. of CHI '09, Boston, USA, April 2009. 